\title{
Envisioning an Ambient Smart Calendar to Support Aging in Place
}

Alexandra Voit

VIS, University of Stuttgart

Stuttgart, Germany

alexandra.voit@vis.uni-

stuttgart.de

Elizabeth Stowell

Northeastern University

Boston, US

stowell.e@husky.neu.edu

Dominik Weber

VIS, University of Stuttgart

Stuttgart, Germany

dominik.weber@vis.uni-

stuttgart.de
Christoph Witte

Intuity Media Lab GmbH

Stuttgart, Germany

witte@intuity.de

\section{Daniel Kärcher}

Intuity Media Lab GmbH

Stuttgart, Germany

kaercher@intuity.de

\section{Niels Henze}

VIS, University of Stuttgart

Stuttgart, Germany

niels.henze@vis.uni-stuttgart.de

Permission to make digital or hard copies of all or part of this work for personal or classroom use is granted without fee provided that copies are not made or distributed for profit or commercial advantage and that copies bear this notice and the full citation on the first page. Copyrights for components of this work owned by others than ACM must be honod. Abstrachg wh credt is permilted. To copy otherwise, or republish to post on servers or to redistribute to lists, requires prior specific permission and/or fee. Request permissions from Permissions@acm.org.

Ubicomp/SWC 16 Adjunct, September 12-16,

DOl: $h$ ttp $/ / /$ dx doi.org/10.1145/2968219.2968555

\begin{abstract}
The world's population is aging, and this shift in demographics will place a high burden on the world's healthcare systems. Smart technology provides a potential method of addressing the demands of these changing demographics. In this paper, we describe the design for a smart calendar that supports elderly people in their daily tasks and activities with the use of ambient notifications. We describe a typical day in the life of a target user, and how the smart calendar system would support the user in living self-sufficiently as she ages. We then discuss design challenges for implementing the persuasive technology. These challenges include correctly detecting a change in behavior and their urgency, the effectiveness of ambient notifications to create health behavior change, and privacy concerns with data collection and sharing.
\end{abstract}

\section{Author Keywords}

Notifications; Attention Management; Aging in Place; Ambient Information System; Smart Home; Internet of Things

\section{ACM Classification Keywords}

H.5.m [Information interfaces and presentation]: Miscellaneous 


\section{Introduction}

The world's population is aging, and this shift in demographics will influence many parts of the society. By 2050 , the United Nations predicts that the percentage of the world's population 60 years or older will grow from 12.3 percent to 21.5 percent [11]. In Europe, this population is predicted to grow from 23.9 to 32.8 percent [11]. This shift in demographics will place a high burden on the healthcare system, with higher demand for medical care associated with aging. We will not have the resources to take care of the aging population within the constraints of our current health care system.

In response to the world's aging population, a lot of focus has been placed on developing assistive technologies to enhance and extend current care for the elderly. Such technologies focus on augmenting current health care services, and also on extending the period of time that an individual can live self-sufficiently [1, 2, 3, 4, 5, 7, 9]. Our vision is to create a persuasive system that supports the elderly who choose to live alone in their homes for longer. We propose a design for a smart calendar that interacts with various smart home devices to support the user in their daily activities and to encourage healthy behavior.

In this paper, we describe how a smart calendar could support elderly people in their daily tasks and activities. To do so, we describe a typical day in the life of a target user, and how the smart calendar system would be integrated into her daily life. Through this scenario, we will describe how the system will support our target user when she is relatively healthy. We will also describe how the system will support the target user when her change in behavior suggests potential illness.

\section{Background}

One of the major challenges of developing assistive technologies for older adults is developing technologies that are acceptable to the user. Much evidence suggests that older adults are willing to incorporate technology into their every day lives [2, 6, 8]. Peek et al. summarized factors that influence the acceptance of technology for aging in place [6] They found six major factors that include concerns about cost and privacy related to technology use, anticipated benefits of the technology, perceived need for the technology, availability of alternatives to technology, the influence of family or friends, and various characteristics of the older adults.

Rashidi and Mihailidis presented design challenges for ambient-assisted living tools for elderly people [7]. They recommend using a simple interface and to avoid parallel tasks in order to minimize the possibility of errors. The user of such systems must have the control over the system and must be able to override automatic processes or decisions. Such systems should be adaptable to the users' needs and the current context. However, the authors suggest to grant access to the system to other stakeholders, such as doctors or family members. Privacy issues should be considered for designing ambient-assistance systems. Consolvo et al. allowed elderly users to decide which information they would like to share. This increased the users' sense of control and trust in the system [1].

In interviews with elderly people living in Europe, Sixsmith et al. found that well-being, and not just illness management, is an important aspect of aging in place [10]. Factors related to well-being included self-sufficiency in daily activities, balancing and maintaining their social lives, and staying active. Rosenberg et al. also found that older adults want technology that would keep them mentally and phys- 


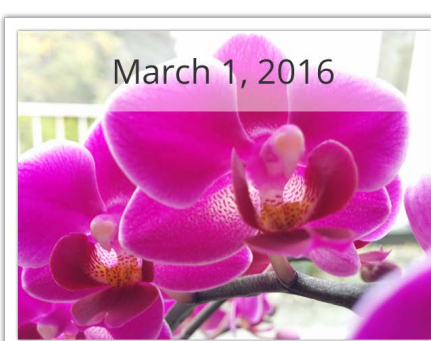

Mo Tu We Th Fr Sa Su

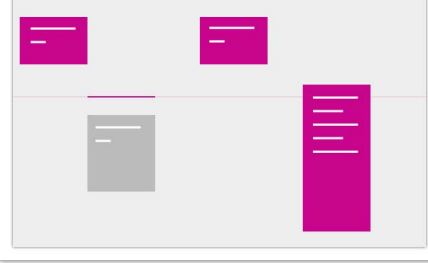

Figure 1: Concept image of the calendar. Suggested events are shown as gray entries. ically active, technology that is simple and has intelligent and automatic functions, technology that can be adapted to the individual user, and technology that is not associated with old age. Demiris et al. investigated possible use-cases for smart home systems for older adults [2]. They found tha potential users desired systems that provide emergency help, reminders about medical appointments, and monitoring of the house (e.g. oven safety controls).

Systems already exist that begin to address some of these concerns. Garcia Vazquez et al. developed an Ambient Information System (AIS) for a smartwatch to support medication adherence among elderly users [3]. Garcia Vazquez et al. then further developed the system for a smartphone [4]. The system uses audio cues and notifications on the smartphone to prompt the users to take medication, and also provides information about dosage and purpose of the medication. The system uses an image of a bird to visualize the user's adherence over the past week, with the intention of encouraging the users to continue taking the medication. Better adherence from the user leads to the bird growing from an egg to an adult who can fly from the nest.

The role of ambient systems in supporting social contact to family members or other care-givers has also been explored. The use of digital portraits to support communication between family members who do not live together is promising $[1,5,9]$. Digital portraits of elders have been used to aid both local and distant family members in caring for aging parents [1, 9]. There, portraits display information about the daily activity of the elderly user that is collected by sensors places in their house. In both studies, caregiver participants reported increased awareness about their elder's daily life.

\section{The smart calendar system}

We envision a smart calender in the context of smart home environments (see Figure 1). The smart calendar will be placed on the wall and provides entirely ambient notifications to the user. Additionally, the smart calendar can also be viewed on the user's smartphone, allowing the user to interact with the calendar on the go.

\section{The scenario}

In this scenario, we describe a typical day of an elderly, retired person. This scenario will be used to explain the functionality of the smart calendar in detail.

Maria Miller is a sixty-eight year old, retired mathematics teacher who has no major health concerns. Maria is divorced, and lives alone in an apartment in the city center of London. Her only child, her daughter, lives with her family a few hours away in Cornwall. In the morning, Maria goes to the market to buy fresh food for the day. Every day, she cooks herself lunch. While she cooks, Maria enjoys listening to classical music. In the evening, Maria typically calls her daughter and speaks with her grandchildren. Every Saturday, Maria meets up with friends to play cards in the afternoon.

\section{Functionality}

The smart calendar supports two different modes. For users who do not have health concerns, the calendar acts as a digital calendar that is both accessible on a smartphone and through its physical representation in the user's smart home. In addition, the calendar suggests events for the user to attend, supports daily activities and tasks, and supports social contact. For users with mental or physical illness, the calendar is able to detect unhealthy changes in behavior, and prompts the user with various types of notifications. 
State: in health

Event suggestions Maria uses her smart calendar as she would use a typical digital calendar. She checks the calendar to not forget her events. She also adds new events as she becomes aware of them or edits details of existing events. Additionally, the calendar automatically makes suggestions for other events that fit free time slots in Maria's calendar. The smart calendar suggests local events that she might enjoy according to her preferences. For example, Maria frequently listens to classical music in the kitchen while cooking. Therefore, the calendar provides information about upcoming classical music concerts in her city.

Support for daily activities and tasks Like many calendar applications, the smart calendar also supports to-do lists. Maria's smart fridge and smart cupboards keep track of what food is available, and what food is about to spoil. When Maria is about to run out of essentials (e.g. coffee or butter), and of toiletries (e.g. shampoo, soap) the smart calendar will propose a shopping event with an automatically generated grocery list. In addition to this, if Maria is planning recipes for the next day on a smart device, the ingredients for the selected recipes are also added to the grocery list. The calendar uses the items from the list to create suggestions for nearby grocery stores, which are open and sell all of the ingredients she requires.

Support for family connection In the evening, Maria often calls her daughter and grandchildren. However, she does not want to call and disrupt dinner time or bed time. To avoid this, Maria is able to synchronize her calendar with her daughter's calendar to find good times to call.

State: in sickness

Missed social events Maria meets her friends every Saturday in a cafe in the city to play cards with them. However in the past few weeks, she has not attended and her friends played cards without her. The meeting with her friends was Maria's main source of social contact in the week. Instead of attending the meetings, she has been staying home alone and watching television. This decline in social interaction may be a result of mental illness, such as depression. Social interaction is important to Maria's well-being. Therefore, to motivate Maria to attend the meetings with her friends again, the calendar displays photographs of the friends she typically plays cards with, as well as images of card games. If Maria still does not meet her friends to play cards, and instead chooses to watch the television, notifications from the calendar will appear on the smart TV screen. These notifications will remind her of the upcoming date and time to meet with her friends.

Missing contact with other family members Like in the scenario where Maria plays cards, it is important for Maria to stay in contact with her family. If Maria goes many days without contacting her family, the calendar will display images of her family members more frequently. If an unusually long time passes with no contact with family members, Maria's family members will receive a notification to check in on Maria.

We envisioned potential functions of a smart calendar. In the future, we plan to build and evaluate such a smart calendar. In addition to the aforementioned themes highlighted by Peek et. al [6], we find the following themes to be crucial to investigate future work.

\section{Event suggestions}

We envision that the smart calendar will provide the user with suggestions for new events. Therefore, the calendar must learn about the user's interests, and how willing the user is to attend different types of events. Certain users 
may respond well to event suggestions that are very different from their typical events, but too many unrelated suggestions may also cause them to ignore or devalue them.

\section{Changes in behavior}

At any point, users may no longer engage in their typical behavior, and ignore ambient cue to action. These behaviors may change for a variety of reasons that do not require intervention and that do not suggest a health concern. What type of change in behavior requires an urgent response remains an open question. The user may no longer play cards with friends, because she has become bored with playing the same game each week. The system must be able to recognize this type of situation and learn the user's new pass times that replace playing cards. Detecting the difference between intentional decisions and illness-induced behavior changes is a major challenge for the envisioned system.

\section{Appropriate reactions on behavior change}

We must investigate the effectiveness of ambient notifications when the system detects alarming changes in the user's behavior. For certain behavior changes, the system may need to notify the user more intrusively. Therefore, the type of notification used must be dependent on the context and type of behavior. For changes which have influence on the user's health, such as ignoring notifications for buying food which will result in hunger, ambient notifications may not be suitable. Furthermore, there may be certain illnesses or stages of illness, e.g. late-stage Alzheimers, for which the smart calendar would be ineffective. For these illnesses, the system will require different types of assistance.

Privacy issues

An important question is how social connections such as family members or close friends should be incorporated into the system. Incorporating family, friends or other stakehold- ers, like doctors, may provide the social support needed for the user to engage in healthy behavior; however, involving these caregivers creates questions related to privacy. It is a challenge to combine the interest of family members for having an awareness of the elderly's activities with privacy concerns of the elderly. Therefore, we must investigate what type of information family members or other stakeholders should have access to.

Consolvo, et al. allowed the elderly user to have control over which information she shared [1]. This requires the user to be aware of the types of data being collected. However for this smart calendar, there must be a compromise between providing caregivers with necessary information and giving the elderly user control over what data can be shared. The public nature of the calendar within the house presents another privacy concern. How can we assure context aware notifications and how will the system change when the elderly has visitors.

\section{Safety issues}

Another challenge is to extend this calendar to encompass medical-related notifications, which will require more critical considerations for the user's safety. If the calendar offers a feature to support medication adherence, the effectiveness of reminder notifications becomes even more important.

Support multi-user functionality

A future consideration for the smart calendar is how to handle multiple people's schedules. Families, couples, or cohabiting people present new challenges for designing a smart calendar, and may require a different interface to handle multiple schedules and notifications.

\section{Summary}

In this paper, we presented our vision of a smart calendar which supports elderly people with their daily tasks and 
activities. We described the calendar's envisioned functionality for healthy people as well as for people with mental or physical illnesses. We then discussed upcoming themes that we plan to investigate in the future, including detecting negative behavior changes, the effectiveness of ambient notifications to create health behavior change, and privacy concerns with data collection and sharing.

Acknowledgments: This work is supported by the German Ministry of Education and Research (BMBF) within the DAAN project (13N13481), by the DFG within the SimTech Cluster of Excellence (EXC 310/1, EXC 310/2) and by the NSF under grant IIA-1358096.

\section{REFERENCES}

1. Sunny Consolvo, Peter Roessler, and Brett E. Shelton. 2004. The CareNet Display: Lessons Learned from an In Home Evaluation of an Ambient Display. Springer Berlin Heidelberg, Berlin, Heidelberg, 1-17.

2. George Demiris, Marilyn J Rantz, Myra A Aud, Karen D Marek, Harry W Tyrer, Marjorie Skubic, and Ali A Hussam. 2004. Older adults' attitudes towards and perceptions of 'smart home' technologies: a pilot study. Medical informatics and the Internet in medicine 29, 2 (2004), 87-94.

3. Juan Pablo Garcia Vazquez, Marcela D Rodriguez, and Angel G Andrade. 2010. Design dimensions of ambient information systems to assist elderly with their activities of daily living. In Proc. UbiComp'10 Adjunct. ACM, 461-464.

4. Juan Pablo García-Vázquez, Marcela D Rodríguez, Ángel G Andrade, and José Bravo. 2011. Supporting the strategies to improve elders' medication compliance by providing ambient aids. Personal and Ubiquitous Computing 15, 4 (2011), 389-397.

5. Eva Lenz, Marc Hassenzahl, Wasili Adamow, Patrick Beedgen, Kirstin Kohler, and Thies Schneider. 2016. Four Stories About Feeling Close Over A Distance. In TEl '16 (TEl '16). ACM, New York, NY, USA, 494-499.

6. Sebastiaan T.M. Peek, Eveline J.M. Wouters, Joost van Hoof, Katrien G. Luijkx, Hennie R. Boeije, and Hubertus J.M. Vrijhoef. 2014. Factors influencing acceptance of technology for aging in place: A systematic review. International Journal of Medical Informatics 83, 4 (2014), 235 - 248.

7. P. Rashidi and A. Mihailidis. 2013. A Survey on Ambient-Assisted Living Tools for Older Adults. IEEE Journal of Biomedical and Health Informatics 17, 3 (May 2013), 579-590.

8. Lena Rosenberg, Anders Kottorp, and Louise Nygård. 2012. Readiness for technology use with people with dementia the perspectives of significant others. Journal of Applied Gerontology 31, 4 (2012), 510-530.

9. Jim Rowan and Elizabeth D. Mynatt. 2005. Digital Family Portrait Field Trial: Support for Aging in Place. In Proc. CHI'05. ACM, New York, NY, USA, 521-530.

10. J. Sixsmith, A. Sixsmith, A. Malmgren Fänge, D. Naumann, C. Kucsera, S. Tomsone, M. Haak, S. Dahlin-Ivanoff, and R. Woolrych. 2014. Healthy ageing and home: The perspectives of very old people in five European countries. Social Science \& Medicine 106 (2014), 1 - 9.

11. UN. 2015. World population aging. (2015). 